\begin{tabular}{|c|l|}
\hline Title & Virtual-state character of the two-body system in the complex scaling method \\
\hline Author(s) & Odsuren, Myagmarjav; Kikuchi, Y uma; Myo, Takay uki; Khuukhenkhuu, Gonchigdorj; Masui, Hiroshi; Kato, Kiy oshi \\
\hline Citation & $\begin{array}{l}\text { Physical review C, 95/6), 064305-1-064305-7 } \\
\text { https://doi.org/10.1103/PhySRevC.95.064305 }\end{array}$ \\
\hline Issue Date & 2017-06-07 \\
\hline Doc URL & http://hdl.handle.net/2115/67008 \\
\hline Rights & ○2017 A merican Physical Society \\
\hline Type & article \\
\hline File Information & PhysRevC95 064305.pdf \\
\hline
\end{tabular}

Instructions for use 


\title{
Virtual-state character of the two-body system in the complex scaling method
}

\author{
Myagmarjav Odsuren, ${ }^{1, *}$ Yuma Kikuchi, ${ }^{2,3, \dagger}$ Takayuki Myo, ${ }^{4,5, \ddagger}$ Gonchigdorj Khuukhenkhuu, ${ }^{1, \S}$ \\ Hiroshi Masui, ${ }^{6, \|}$ and Kiyoshi Katō ${ }^{7, \mathbb{I}}$ \\ ${ }^{1}$ School of Engineering and Applied Sciences, Nuclear Research Center, National University of Mongolia, Ulaanbaatar 210646, Mongolia \\ ${ }^{2}$ Department of Physics, Osaka City University, Osaka 558-8585, Japan \\ ${ }^{3}$ RIKEN Nishina Center, Wako 351-0198, Japan \\ ${ }^{4}$ General Education, Faculty of Engineering, Osaka Institute of Technology, Osaka 535-8585, Japan \\ ${ }^{5}$ Research Center for Nuclear Physics (RCNP), Osaka University, Ibaraki 567-0047, Japan \\ ${ }^{6}$ Information Processing Center, Kitami Institute of Technology, Kitami 090-8507, Japan \\ ${ }^{7}$ Nuclear Reaction Data Centre, Faculty of Science, Hokkaido University, Sapporo 060-0810, Japan
}

(Received 17 April 2017; published 7 June 2017)

\begin{abstract}
In association with the property of the $1 / 2^{+}$state observed just above the ${ }^{8} \mathrm{Be}\left(0^{+}\right)+n$ threshold energy in ${ }^{9} \mathrm{Be}$, we investigate the photodisintegration cross section of an $s$ state in a simple schematic two-body model using the complex scaling method. The photodisintegration cross section, continuum level density, scattering phase shifts, and scattering length are discussed in relation with the virtual state. These scattering observables show strong divergences when a virtual state is located near the physical scattering region.
\end{abstract}

DOI: 10.1103/PhysRevC.95.064305

\section{INTRODUCTION}

The peculiar behavior of the cross sections near the threshold energy region has been of great interest for a long time. Previously, a large photodisintegration cross section of the deuteron has been observed, and its reaction mechanism has been discussed in association with nuclear interactions [1]. Recently, the Coulomb breakup reaction cross sections of neutron halo nuclei have been investigated to understand the mechanism of the low-lying enhancement in the cross section in relation with exotic nuclear halo structure [2]. Furthermore, from an astrophysical viewpoint, much interest has been concentrated on the low-energy cross section of the ${ }^{9} \mathrm{Be}$ photodisintegration, in which the ${ }^{9} \mathrm{Be}$ nucleus breaks up into the $\alpha+\alpha+n$ three-body final state [3].

For these problems, we have tried to understand the origin of the peaks in cross sections observed near the threshold energies from the viewpoint of nuclear many-body resonances. In order to describe the many-body resonances explicitly, we employed the complex scaling method (CSM), which provides us with a promising way to obtain the resonant states similarly to boundstate calculations. In our previous work [4], we discussed that the observed peak in the ${ }^{9} \mathrm{Be}$ photodisintegration above the ${ }^{8} \mathrm{Be}+n$ threshold can be well explained as a result of a ${ }^{8} \mathrm{Be}+$ $n$ two-body virtual state but not of an $\alpha+\alpha+n$ three-body resonance.

A virtual-state character on light nuclei including halo nuclei has been studied using different theoretical methods, namely, the analytical continuation method [5] and the Jost function method [6]. We use the CSM [7-10] to investigate

\footnotetext{
*odsuren@seas.num.edu.mn

†yuma@sci.osaka-cu.ac.jp

takayuki.myo@oit.ac.jp

§khuukhenkhuu@seas.num.edu.mn

"hgmasui@mail.kitami-it.ac.jp

Ikato@nucl.sci.hokudai.ac.jp
}

a virtual state in this work. Generally, the CSM plays an important role not only in the investigation of many-body resonances but also in the description of nonresonant manybody continuum states [11-16]. In the CSM, the resonant state is obtained as the pole of the $S$ matrix in the fourth quadrant of the complex momentum plane, but the virtual state cannot be obtained directly as an isolated energy-eigenvalue because the virtual pole lies on the negative energy axis of the second Riemann sheet corresponding to the negative imaginary momentum axis, which is a border of the fourth quadrant.

As far as we know, there is no previous study (except our previous one [4]) where the CSM can be applied successfully to virtual state. Applying the CSM to the $\alpha+\alpha+n$ three-body model for ${ }^{9} \mathrm{Be}$, we have shown that the sharp peak of the photodisintegration cross section experimentally observed just above the ${ }^{8} \mathrm{Be}\left(0^{+}\right)+n$ threshold is explained as a $1 / 2^{+}$virtual state of the ${ }^{8} \mathrm{Be}\left(0^{+}\right)+n$ two-body configuration. However, this result has not been obtained from the virtual-state solution directly, and the virtual-state properties, such as pole position and scattering length, have not be clarified. To obtain a virtual-state pole, the analytical continuation method seems to the most available one. But, recently, Kamimura et al. [17] discussed that sometimes the analytical continuation method does give accurate pole positions for three-cluster systems. It is strongly desired to develop the CSM in order to obtain the direct information of virtual states and also broad resonance states.

The purpose of this work is to investigate, in the framework of the CSM, the structure of a virtual state in an $s$-wave using a simple schematic two-body model which simulates the ${ }^{8} \mathrm{Be}\left(0^{+}\right)+n$ system. We discuss the virtual state in detail, calculating the photodisintegration cross section corresponding to the existence of a virtual state. We show that when a virtual state approaches the zero energy near the physical scattering region, it has a strong influence on the scattering observables. In our previous work of ${ }^{9} \mathrm{Be}$ [4], we concluded that the virtual state is responsible for the enhancement of the 
photodisintegration cross section near the two-body ${ }^{8} \mathrm{Be}+n$ threshold. In the present work, we confirm that the virtual state leads to rapid variations in phase shifts and in scattering lengths as changing the potential strengths. Increasing the potential strength, the virtual state is moving away from the scattering region into the bound-state region and its effects on the scattering observables will emerge in the cross section, the phase shifts, and scattering length. We calculate those quantities by using the level density obtained in the CSM. We carefully investigate the behavior of scattering observables in relation to the existence of the virtual state located near the threshold by different potential strengths.

In Sec. II, we explain our framework, applying the CSM to a simple schematic two-body model. In Sec. III, we give the obtained results and discussions. Finally, the conclusion is given in Sec. IV.

\section{FORMALISM}

\section{A. Complex scaling method}

To understand the mechanism of a low-energy peak formation in the photodisintegration of the ${ }^{9} \mathrm{Be}\left(1 / 2^{+}\right)$state, we investigate a simple schematic two-body model corresponding to the ${ }^{8} \mathrm{Be}+n$ structure in ${ }^{9} \mathrm{Be}$. In this schematic model, both clusters are assumed to be structureless and spinless, and relative motion between clusters is described by the following Schrödinger equation:

$$
H \Psi_{J^{\pi}}^{v}=E_{\nu} \Psi_{J^{\pi}}^{\nu}
$$

where $J^{\pi}$ is the spin and parity and $v$ is the state index. The Hamiltonian $H$ consists of the relative kinetic energy $T=$ $-\frac{\hbar^{2}}{2 \mu} \nabla^{2}$ and the potential $V$ for relative motion, where $\mu$ is the reduced mass. Then the Hamiltonian is given as

$$
H=-\frac{\hbar^{2}}{2 \mu} \nabla^{2}+V(r),
$$

where we assume the simple Gaussian potential

$$
V(r)=V_{1} \exp \left(-a r^{2}\right)
$$

In this model, for simplicity, we put $\frac{\hbar^{2}}{2 \mu}=1\left(\mathrm{MeV} \mathrm{fm}^{2}\right)$. This potential was introduced in Ref. [18] by taking the first term of the potential with an $a=0.16 \mathrm{fm}^{-2}$. The potential depth $V_{1}$ is adjusted to reproduce one bound state of $s$ and $p$ waves. The first $s$-wave bound state is considered the Pauli-forbidden state in order to simulate the ${ }^{8} \mathrm{Be}+n$ system, and the bound $p$-wave solution is the ground state corresponding to the $3 / 2^{-}$ state of ${ }^{9} \mathrm{Be}$. We calculate the electric dipole $(E 1)$ transition from the ground $p$-wave state to the excited unbound $s$-wave states.

The eigenvalue problem of Eq. (1) is generally solved under a boundary condition of asymptotic outgoing waves for bound and unbound states. The enforcement of the boundary condition directly enables us to obtain bound states in an $L^{2}$ functional basis set because the states have negative energies and a damping behavior in the asymptotic region. The unbound states are defined as the eigenstates belonging to the complex eigenenergy which corresponds to a complex momentum value in the lower half plane (unphysical plane $[9,10]$ ).
The CSM has been introduced to obtain resonant states within $L^{2}$ basis functions and is defined by the following complex dilation transformation for relative coordinate $\vec{r}$ and momentum $\vec{k}$ [19]:

$$
\vec{r} \rightarrow \vec{r} e^{i \theta}, \quad \vec{k} \rightarrow \vec{k} e^{-i \theta},
$$

where $\theta$ is a scaling angle given by a real number and $0<\theta<\theta_{\max }$. The maximum value $\theta_{\max }$ is determined to keep analyticity of the transformed potential. For example, $\theta_{\max }=\pi / 4$ for a Gaussian potential. In a many-body system, this transformation makes every branch cut rotated by $-2 \theta$ from the real axis on the complex energy plane. Applying this transformation to Eq. (1), we obtain the complex-scaled Schrödinger equation

$$
H^{\theta} \Psi_{J^{\pi}}^{\nu}(\theta)=E_{\nu}^{\theta} \Psi_{J^{\pi}}^{\nu}(\theta)
$$

The complex-scaled Hamiltonian $H^{\theta}$ and wave function $\Psi_{J^{\pi}}^{\nu}(\theta)$ are defined as $U(\theta) H U(\theta)^{-1}$ and $U(\theta) \Psi_{J^{\pi}}^{\nu}$, respectively, and see Refs. [9,10] for details.

Applying the $L^{2}$ basis function method, the radial wave function is expanded as

$$
\Psi_{J^{\pi}}^{v}(\theta)=\sum_{n=1}^{N} c_{n}^{J^{\pi} v}(\theta) \phi_{n}(r),
$$

where $\phi_{n}(r)$ is an appropriate basis function set. The expansion coefficients $c_{n}^{J^{\pi} v}$ and the complex energy eigenvalues $E_{v}^{\theta}$ are obtained by solving the complex-eigenvalue problem given in Eq. (5). The complex energies of resonant states are obtained as $E_{r}=E_{r}^{\mathrm{res}}-i \Gamma_{r} / 2$, when $\tan ^{-1}\left(\Gamma_{r} / 2 E_{r}^{\mathrm{res}}\right)<2 \theta$.

\section{B. Continuum level density and scattering phase shift}

The continuum level density, $\Delta(E)$, is defined as a function of the real energy $E[20,21]$

$$
\Delta(E)=-\frac{1}{\pi} \operatorname{Im}\left\{\operatorname{Tr}\left[G^{+}(E)-G_{0}^{+}(E)\right]\right\},
$$

where

$$
G^{+}(E)=(E+i \epsilon-H)^{-1}, \quad G_{0}^{+}(E)=\left(E+i \epsilon-H_{0}\right)^{-1}
$$

are the full and free Green's functions for $H$ of Eq. (2) and $H_{0}=-\frac{\hbar^{2}}{2 \mu} \nabla^{2}$, respectively. The continuum level density is also related to the scattering phase shift $\delta(E)$ and the relation is expressed in a single channel case as $[20,21]$ :

$$
\Delta(E)=\frac{1}{\pi} \frac{d \delta(E)}{d E} .
$$

Using Eqs. (7) and (8), we can obtain the phase shift $\delta(E)$ in terms of the eigenvalues of $H$ (with interaction) and $H_{0}$ (without interaction) by integrating the continuum level density over the energy $E$. When we apply the CSM and obtain the complex-scaled Green's function, the continuum level density can be expressed as

$$
\Delta_{\theta}(E)=-\frac{1}{\pi} \operatorname{Im}\left\{\operatorname{Tr}\left[\frac{1}{E-H^{\theta}}-\frac{1}{E-H_{0}^{\theta}}\right]\right\} .
$$

In the calculation of $\Delta_{\theta}(E)$ in Eq. (9), we apply the extended completeness relation whose detailed explanation is given 
in Refs. [22-24]. Here, we use the solutions obtained by diagonalization of matrix elements of $H^{\theta}$ and $H_{0}^{\theta}$ with a finite number $N$ of basis functions in Eq. (6). The energy eigenvalues of $H_{0}^{\theta}$ are given as $\epsilon_{k}^{0 r}-i \epsilon_{k}^{0 i}$ with the label $k=1, \ldots, N$. All of these values are distributed on the " $2 \theta$ line." The continuum level density of Eq. (7) is approximated as

$$
\begin{aligned}
\Delta(E) \approx & \Delta_{\theta}^{N}(E)=-\frac{1}{\pi} \operatorname{Im}\left[\sum_{b=1}^{N_{b}} \frac{1}{E+i 0-E_{b}}\right. \\
& +\sum_{r=1}^{N_{r}^{\theta}} \frac{1}{E-E_{r}^{\mathrm{res}}+i \Gamma_{r} / 2}+\sum_{c=1}^{N_{c}^{\theta}} \frac{1}{E-\epsilon_{c}^{r}+i \epsilon_{c}^{i}} \\
& \left.-\sum_{k=1}^{N} \frac{1}{E-\epsilon_{k}^{0 r}+i \epsilon_{k}^{0 i}}\right],
\end{aligned}
$$

where the eigenvalues $E_{v}^{\theta}$ are classified into those of $N_{b}$ bound states, $N_{r}^{\theta}$ resonant states, $N_{c}^{\theta}$ continuum states, and totally $N=N_{b}+N_{r}^{\theta}+N_{c}^{\theta}$. It is important to note that the approximated continuum level density, $\Delta_{\theta}^{N}(E)$, has a dependence on the scaling angle $\theta$ substantially because we employ a finite number $N$ of the basis states to expand the complex scaled wave functions. In the calculation we adopt a sufficiently large number of $N$ to keep the numerical accuracy and to make the $\theta$ dependence negligible in the solutions [25].

We can also calculate the phase shift from $\Delta_{\theta}^{N}(E)$

$$
\begin{aligned}
\delta_{\theta}^{N}(E)= & N_{b} \pi+\sum_{r=1}^{N_{r}^{\theta}}\left\{-\cot ^{-1}\left(\frac{E-E_{r}^{\mathrm{res}}}{\Gamma_{r} / 2}\right)\right\} \\
& +\sum_{c=1}^{N_{c}^{\theta}}\left\{-\cot ^{-1}\left(\frac{E-\epsilon_{c}^{r}}{\epsilon_{c}^{i}}\right)\right\} \\
& -\sum_{k=1}^{N}\left\{-\cot ^{-1}\left(\frac{E-\epsilon_{k}^{0 r}}{\epsilon_{k}^{0 i}}\right)\right\},
\end{aligned}
$$

where we assume $E \geqslant 0$ [26].

\section{Transition strength and cross section}

Using the energy eigenvalues and eigenstates of the complex-scaled Hamiltonian $H^{\theta}$ and their biorthogonal states of $\left\{\Psi_{J \pi}^{v}(\theta)\right\}$ and $\left\{\tilde{\Psi}_{J \pi}^{v}(\theta)\right\}$, we define the complex-scaled Green's function $\mathcal{G}^{\theta}\left(E ; \xi, \xi^{\prime}\right)$ as

$$
\mathcal{G}^{\theta}\left(E ; \boldsymbol{\xi}, \boldsymbol{\xi}^{\prime}\right)=\left\langle\boldsymbol{\xi}\left|\frac{1}{E-H^{\theta}}\right| \boldsymbol{\xi}^{\prime}\right\rangle=\sum_{v} \frac{\left|\Psi_{J^{\pi}}^{v}(\theta)\right\rangle\left\langle\tilde{\Psi}_{J^{\pi}}^{v}(\theta)\right|}{E-E_{v}^{\theta}}
$$

In the derivation of the right-hand side of Eq. (12), we use the extended completeness relation [22-24]. In the complexscaled Green's function of Eq. (12), outgoing boundary conditions are taken into account by the imaginary parts of the energy eigenvalues $E_{v}^{\theta}$. Using the complex-scaled Green's function, we calculate the photodisintegration cross section.
The cross sections for the electric dipole transitions $\sigma_{E 1}^{\gamma}$ is expressed as the following form:

$$
\sigma_{E 1}^{\gamma}\left(E_{\gamma}\right)=\frac{16 \pi^{3}}{9}\left(\frac{E_{\gamma}}{\hbar c}\right) \frac{d B\left(E 1, E_{\gamma}\right)}{d E_{\gamma}},
$$

where $E_{\gamma}$ is the incident photon energy and $B(E 1)$ is an electric dipole transition strength. Using the CSM and the complex-scaled Green's function Eq. (12), the electric dipole transition strength is given as

$$
\begin{aligned}
\frac{d B\left(E 1, E_{\gamma}\right)}{d E_{\gamma}}= & -\frac{1}{\pi} \frac{1}{2 J_{\mathrm{gs}}+1} \\
& \times \operatorname{Im}\left[\sum_{\nu}\left(\tilde{\Psi}_{J^{\pi}}^{\mathrm{gs}}\left\|\left(\hat{O}^{\theta}\right)^{\dagger}(E 1)\right\| \Psi_{J^{\pi}}^{v}(\theta)\right\rangle\right. \\
& \left.\times \frac{1}{E-E_{v}^{\theta}}\left(\tilde{\Psi}_{J^{\pi}}^{v}(\theta)\left\|\hat{O}^{\theta}(E 1)\right\| \Psi_{J^{\pi}}^{\mathrm{gs}}\right\rangle\right],
\end{aligned}
$$

where $J_{\mathrm{gs}}$ and $\Psi_{\mathrm{gs}}$ represent the total spin and the wave function of the ground state, respectively, and $\hat{O}(E 1)$ is an electric dipole transition operator. The energy $E$ is related to $E_{\gamma}$ as $E=E_{\gamma}-E_{\mathrm{gs}}$, where $E_{\mathrm{gs}}$ is the binding energy of the ground state measured from the threshold.

\section{RESULTS AND DISCUSSIONS}

\section{A. Two-body model for the ${ }^{8} \mathrm{Be}+\boldsymbol{n}$ system}

It is believed that the CSM cannot describe virtual states corresponding to $S$-matrix poles on the negative imaginary axis on the complex momentum plane. In Fig. 1, the mathematical trajectories of $s$-wave $S$-matrix poles are displayed in momentum and in energy planes [27]. In the CSM, eigenvalues on the solid line can be obtained, but those of the broken line cannot be obtained. Then the virtual-state term does not explicitly appear in the continuum level density of Eq. (10) and the phase shift of Eq. (11). However, in our previous work [4], the peak of the photodisintegration cross section calculated by the CSM suggests the existence of the virtual state.

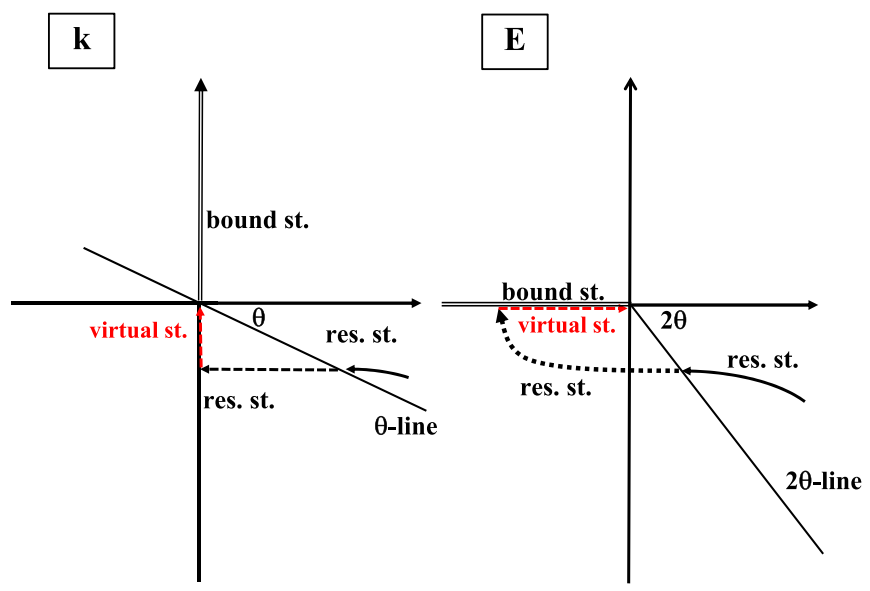

FIG. 1. Trajectories of the $s$-wave poles in momentum $(k)$ and in energy $(E)$ planes. 

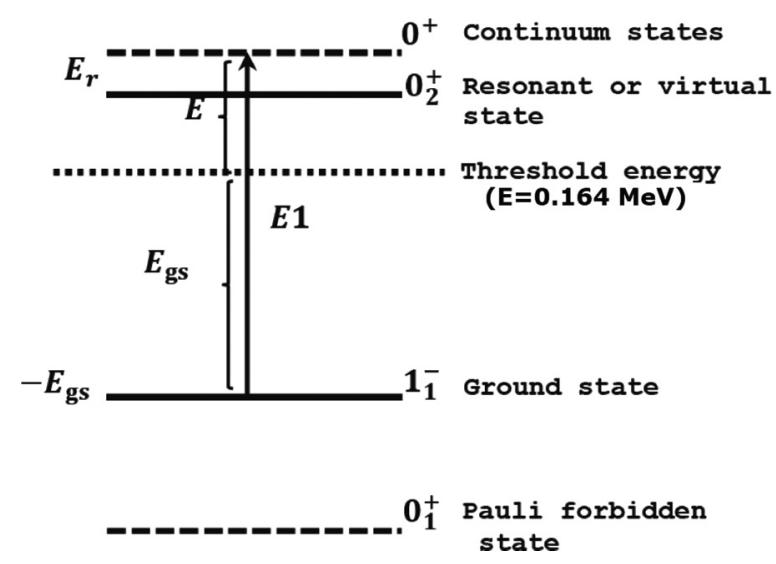

FIG. 2. The energy level diagram of the two-body potential model simulating ${ }^{9} \mathrm{Be}$. The dotted line represents the threshold energy.

To confirm that continuum solutions of the CSM describe the virtual state, we calculate the phase shifts of the $s$ wave using Eq. (11). We solve the eigenvalue problem of Eq. (5) employing the Gaussian basis functions [28] given as

$$
\phi_{i}(r)=N_{\ell}\left(b_{i}\right) r^{\ell} \exp \left(-\frac{1}{2 b_{i}^{2}} r^{2}\right) Y_{\ell m}(\hat{r}),
$$

where the range parameters are given by a geometric progression as $b_{i}=b_{0} \gamma^{i-1} ; i=1, \ldots, N$, and $N_{\ell}\left(b_{i}\right)$ is the normalization factor. We take $N=60$ and employ the optimal values of $b_{0}$ and $\gamma$ to obtain stationary solutions. All results are obtained with $\theta=15^{\circ}$.

The energy level diagram of the potential model is shown in Fig. 2. Here we show the levels of $J^{\pi}=0^{+}$and $1^{-}$, which are obtained by solving the complex-scaled Schrödinger equation. The potential strength $V_{1}$ in Eq. (3) is taken to reproduce one bound $J^{\pi}=0_{1}^{+}$of $s$ waves. But this $J^{\pi}=0_{1}^{+}$solution is assumed to be the Pauli forbidden state, because in this model we simulate the ${ }^{8} \mathrm{Be}\left(0^{+}\right)+n$ system which has the Pauli-forbidden $(0 s)$ neutron configuration. In this model, the $1^{-}$solution describes the ground state. We investigate $E 1$ transitions from the $1^{-}$ground state to the excited $0^{+}$unbound states including the $0_{2}^{+}$state. We investigate the behavior of the $E 1$ transition strength as changing the potential strength $V_{1}$ for $0^{+}$and $1^{-}$states.

The pole trajectories for the $J^{\pi}=0_{1}^{+}, 1^{-}$and $0_{2}^{+}$bound states are shown in Fig. 3. From the results, we can see that the $0_{2}^{+}$state changes from bound to unbound in the range of $-1.45 \mathrm{MeV}<V_{1}<-1.42 \mathrm{MeV}$. For $V_{1}=-1.43 \mathrm{MeV}$, we obtain the bound $0_{2}^{+}$state, but this bound-state solution disappears at $V_{1}=-1.42 \mathrm{MeV}$. The unbound state for $V_{1}=$ $-1.42 \mathrm{MeV}$ is expected to be a virtual state from the $s$-wave pole trajectory shown in Fig. 1.

Using the calculated eigenvalues including the continuum states for $-1.42 \mathrm{MeV}$, we calculate the photodisintegration cross section due to the $E 1$ transition. The result is shown in Fig. 4. It is seen that the peak is obtained at a lower energy region with the similar shape as observed in the ${ }^{9} \mathrm{Be}\left(1 / 2^{+}\right)$ state (shown in Fig. 1 of Ref. [4]).

To understand the virtual-state contribution to the cross section in the CSM calculation more clearly, we calculate the

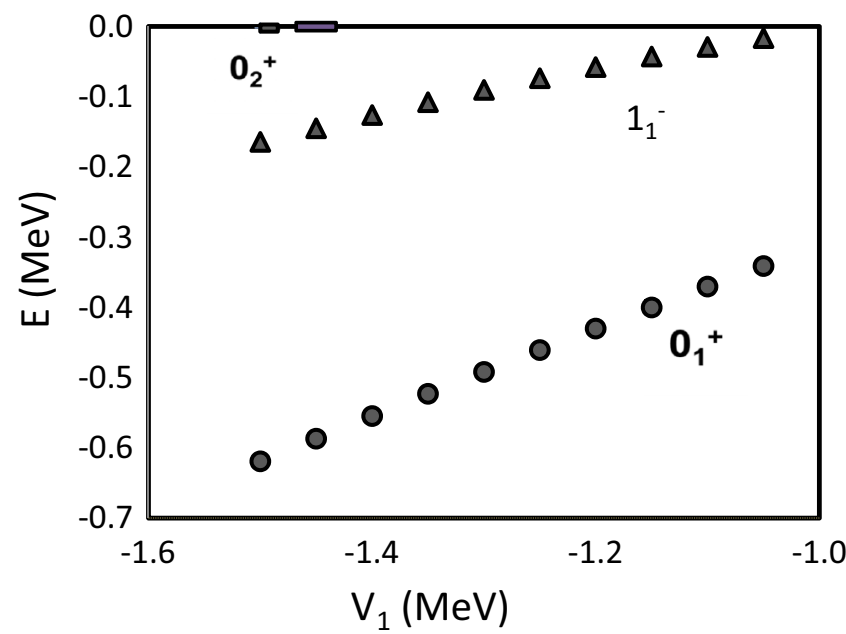

FIG. 3. The pole trajectories for the $J^{\pi}=0_{1}^{+}, 1^{-}$, and $0_{2}^{+}$states. The circles, triangles, and squares display the eigenvalues of the $J^{\pi}=0_{1}^{+}, 1^{-}$, and $0_{2}^{+}$states, respectively, calculated as changing the potential strength $V_{1}$.

continuum level density of the $J^{\pi}=0^{+}$state, using Eq. (10) with $V_{1}=-1.42 \mathrm{MeV}$ and $-1.43 \mathrm{MeV}$, which correspond to virtual-state and bound-state cases, respectively. In Fig. 5, we show the calculated continuum level density. The results show a very similar behavior for $V_{1}=-1.42 \mathrm{MeV}$ and $-1.43 \mathrm{MeV}$, and the difference seems very small except for a small energy region.

To see a difference between the calculated continuum level densities for $V_{1}=-1.42 \mathrm{MeV}$ and $-1.43 \mathrm{MeV}$, we calculate the phase shifts by using Eq. (11). The results are presented in Fig. 6, and we can see a large difference in the low-energy region. In the case of $V_{1}=-1.43 \mathrm{MeV}$, there is one bound state except for the lowest bound state assigned to the Pauli forbidden state, and then the phase shift starts from $\pi$ at

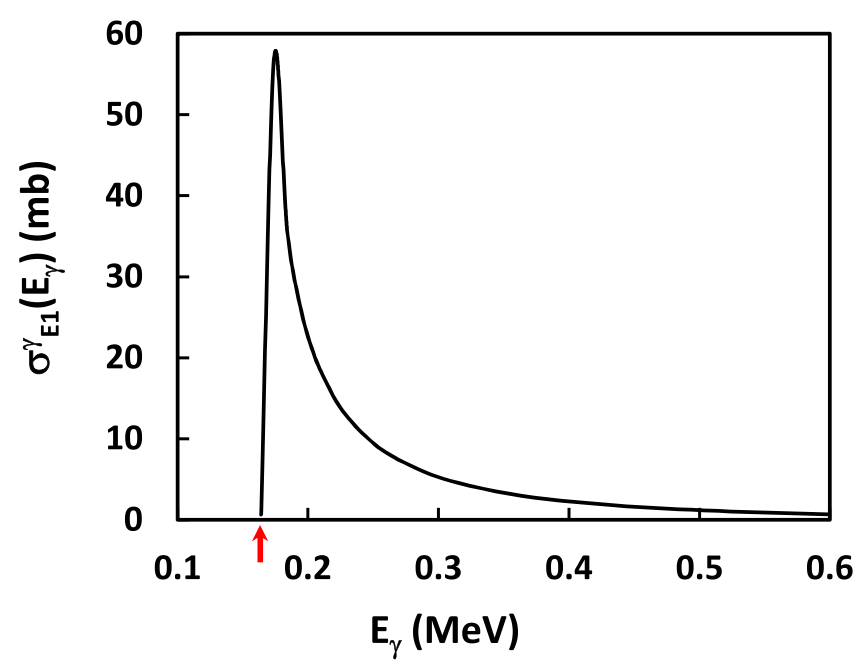

FIG. 4. Photodisintegration cross sections due to the $E 1$ transition calculated with the two-body potential model with the strength $V_{1}=-1.42 \mathrm{MeV}$. The arrow indicates the threshold energy which is shown in the Fig. 2 as a dotted line. 


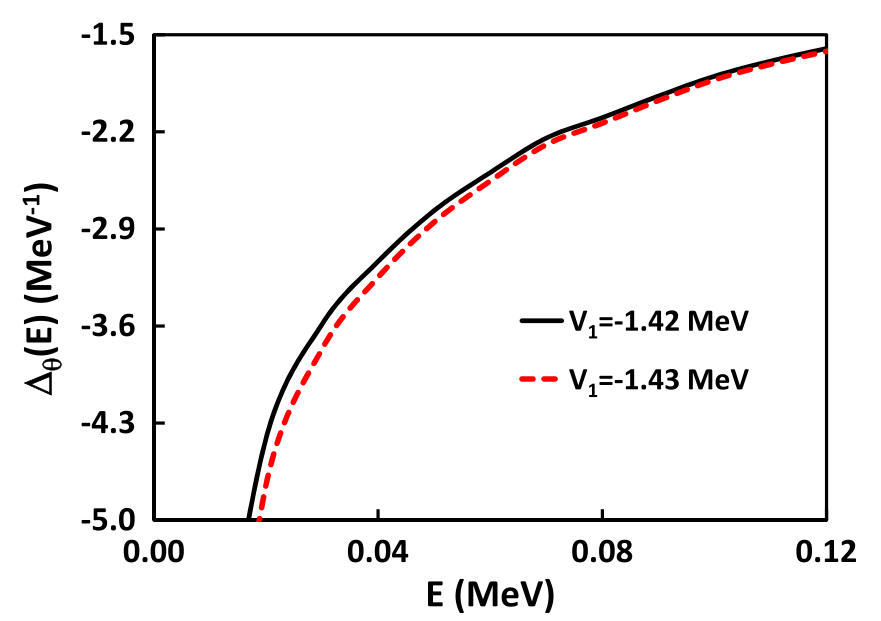

FIG. 5. Calculated continuum level density $\Delta_{\theta}^{N}(E)$ at the strengths of $V_{1}=-1.42 \mathrm{MeV}$ and $-1.43 \mathrm{MeV}$. The black solid and red dashed curves are the results at $V_{1}=-1.42 \mathrm{MeV}$ and $-1.43 \mathrm{MeV}$, respectively.

$E=0 \mathrm{MeV}$ because of the Levinson theorem and decreases with energy. On the other hand, in the case of $V_{1}=$ $-1.42 \mathrm{MeV}$, the phase shift starts from zero and increases up to about $\pi / 3$ but not $\pi / 2$ unlike a resonance. This phase shift behavior supports the appearance of the virtual state. In the case of $V_{1}=-1.43 \mathrm{MeV}$, the phase shift decreases passing across $\pi / 2$ from above, and an enhancement of the cross section due to $\delta=\pi / 2$ is often called an echo.

Furthermore, we calculate the scattering length from the obtained $s$-wave phase shift using the relation

$$
a_{s}=-\lim _{k \rightarrow 0} \tan \delta_{\theta}^{N}(E) / k,
$$

where $k=\sqrt{2 \mu E} / \hbar$ is a momentum. For different potential strengths in the range of $-1.43<V_{1}<-1.42 \mathrm{MeV}$, the calculated scattering lengths $a_{s}$ are shown in Fig. 7. We

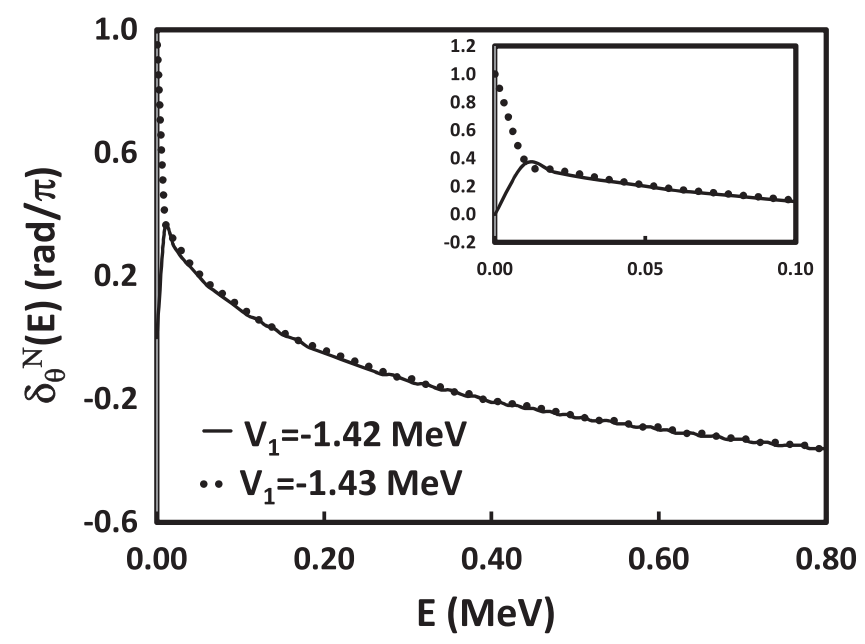

FIG. 6. Calculated phase shifts of the $0^{+}$state for $V_{1}=$ $-1.42 \mathrm{MeV}$ and $-1.43 \mathrm{MeV}$. The solid and open solid curves are phase shifts calculated at $V_{1}=-1.42 \mathrm{MeV}$ and $-1.43 \mathrm{MeV}$, respectively. The scale of the graph was magnified in the inset.

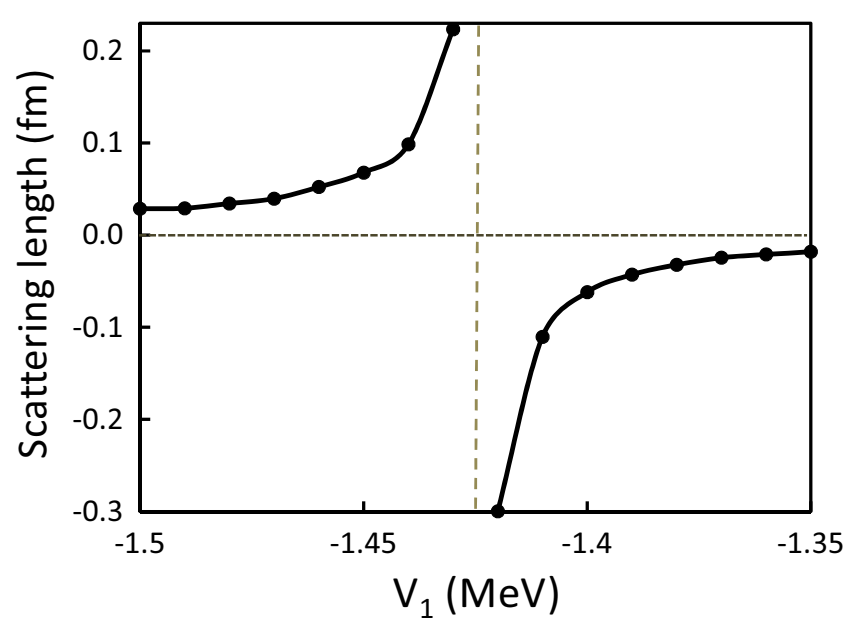

FIG. 7. Scattering length of the $0^{+}$state calculated for $V_{1}=$ $-1.2 \mathrm{MeV}$ and $-1.5 \mathrm{MeV}$ in the CSM. The horizontal dotted line indicates $a_{s}=0$ and the vertical broken line shows a border where the $a_{s}$ changes sign.

find a sudden change of $a_{s}$ in the range of $-1.43<V_{1}<$ $-1.42 \mathrm{MeV}$. While $a_{s}$ is positive for the potential strength $\left(V_{1} \leqslant-1.43 \mathrm{MeV}\right)$ reproducing a bound $0_{2}^{+}$state, $a_{s}$ is negative for $V_{1} \geqslant-1.42 \mathrm{MeV}$. And at $V_{1}=-1.42 \mathrm{MeV}$, it is seen that $a_{s}$ has a large negative value, which also indicates the existence of the virtual state.

From the result of phase shifts which are calculated using the complex-scaled energy eigenvalues, we confirm that the virtual state is included in the continuum solutions of the CSM though it is not an isolated solution. We try to extract a more detailed information on the virtual state, such as the pole position, from the CSM solutions in the next analysis.

\section{B. Virtual state in the CSM}

As we discussed in Ref. [4], the contribution of the virtual state to the cross section is scattered into the continuum $0^{+}$solutions obtained on the rotated $2 \theta$ line in the CSM. Analyzing contributions from each eigenstate on the $2 \theta$ line to the phase shift, we can confirm that the virtual state is not described by a particular one among the continuum solutions. Then, we develop a new method to extract the virtual-state information from the continuum solutions.

The continuum level density $\Delta_{\theta}^{N}(E)$ given by Eq. (10) depends on the potential strength $V_{1}$ and we express this quantity as $\Delta_{\theta}^{N}\left(E ; V_{1}\right)$ for convenience. The quantity $\Delta_{\theta}^{N}\left(E ; V_{1}\right)$ of $J^{\pi}=0^{+}$states is expected to have a contribution from the virtual state in the case of $V_{1}=-1.42 \mathrm{MeV}$, which disappears in the case of $V_{1}=-1.43 \mathrm{MeV}$. In the case of $V_{1}=$ $-1.43 \mathrm{MeV}$, a bound $0^{+}$state appears instead of the virtual state, and then the continuum level density is expressed as

$$
\Delta_{\theta}^{N}(E ;-1.43 \mathrm{MeV})=\Delta_{b}^{2}(E)+\Delta_{c}^{N-2}(E ;-1.43 \mathrm{MeV}),
$$

where $\Delta_{b}^{2}$ and $\Delta_{c}^{N-2}$ are the contribution from bound and continuum states, respectively. In this case, there are two bound $0^{+}$states including the Pauli forbidden state, and then 


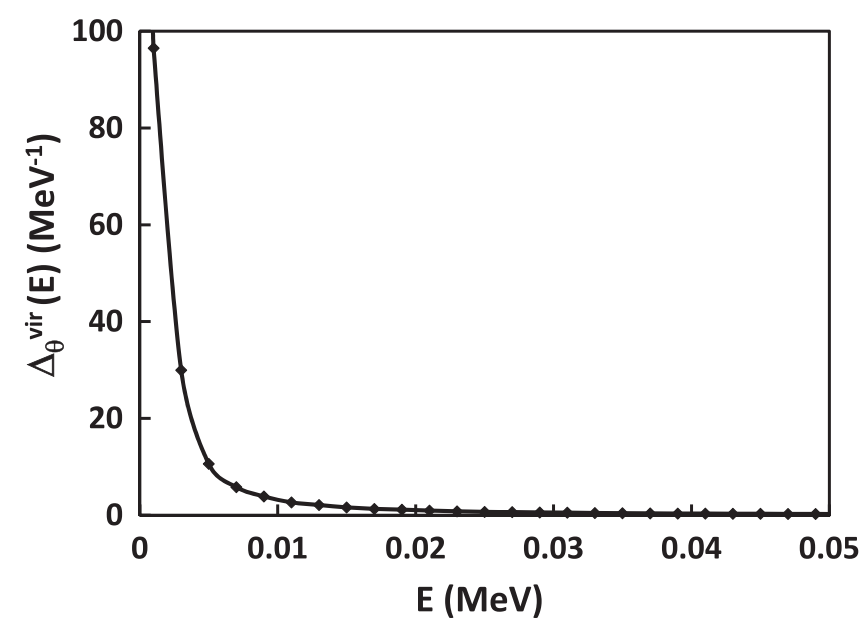

FIG. 8. The difference between the continuum level densities for $V_{1}=-1.42 \mathrm{MeV}$ and $-1.43 \mathrm{MeV}$. Ideally, the continuum level density calculated by the virtual state $\Delta_{\theta}^{\text {virt }}(E)$.

the contribution from continuum states is calculated by using the number of $N-2$ continuum solutions on the $2 \theta$ line in the complex energy plane because of no resonances. In the case of $V_{1}=-1.42 \mathrm{MeV}$, we have

$$
\Delta_{\theta}^{N}(E ;-1.42 \mathrm{MeV})=\Delta_{b}^{1}(E)+\Delta_{c}^{N-1}(E ;-1.42 \mathrm{MeV}),
$$

where the bound $0^{+}$state is the Pauli forbidden state alone.

We calculate a difference

$$
\Delta^{\mathrm{virt}}(E)=\Delta_{c}^{N-1}(E ;-1.42 \mathrm{MeV})-\Delta_{c}^{N-2}(E ;-1.43 \mathrm{MeV})
$$

From this quantity, it is expected to extract the effect of the virtual state on the continuum level density. The difference is displayed in Fig. 8, which shows the sharp peak near the zero energy. Here we assume that $\Delta_{c}^{N-1}(E ;-1.42 \mathrm{MeV})$ consists of two types of contributions; one is a virtual-state contribution and another is a background. The background contribution is also assumed to have a weak dependence of the strength of $V_{1}$. Then the background contributions are considered to be almost the same in both cases of $V_{1}=-1.42 \mathrm{MeV}$ and $-1.43 \mathrm{MeV}$. When the background is expressed by $\Delta_{c}^{N-2}(E ;-1.43 \mathrm{MeV})$ approximately, we can consider that $\Delta^{\text {virt }}(E)$ corresponds to the virtual-state contribution of the continuum level density.

Since the phase shift is obtained by integrating the continuum level density, the phase shift of the virtual state is given as

$$
\delta^{\mathrm{virt}}(E)=\pi \int_{0}^{E} \Delta^{\mathrm{virt}}\left(E^{\prime}\right) d E^{\prime} .
$$

Using the result of $\Delta^{\text {virt }}(E)$ shown in Fig. 8, we obtained the corresponding phase shift as shown in Fig. 9. This result indicates a characteristic behavior of the phase shift of the virtual state. From Eq. (11), it is seen that a bound state gives a constant shift of $\pi$ in the phase shift, and a resonant state gives a monotonic increasing function up to $\pi$ for energy $E$ as discussed in Refs. [4,26]. The phase shift of the virtual state is described by an increasing function of energy

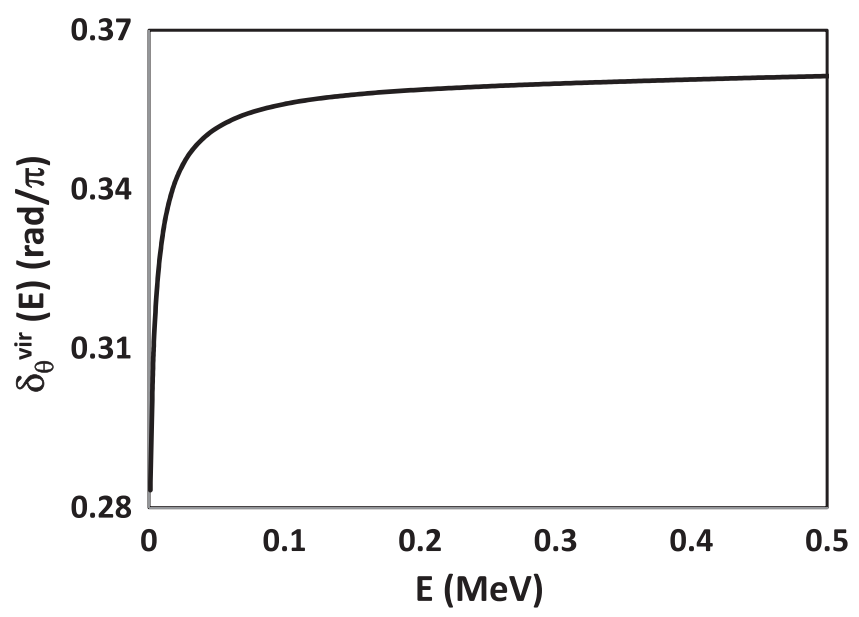

FIG. 9. The phase shift of the virtual state, calculated from the virtual-state continuum level density $\Delta^{\text {virt }}(E)$.

but does not reach $\pi / 2$. The phase shift given in Fig. 6 is understood as a summation of phase shifts of the virtual state and the background states. The background phase shift seems to behave like a monotonic decreasing function of energy, similarly to hard-sphere scattering, as seen from Fig. 6.

The phase shift of the virtual state is also considered to yield from a corresponding pole of the virtual state. Although such a pole cannot be obtained as an eigenvalue solution in the CSM, it is expected to extract the pole position $E_{v}$ on the second Riemann sheet of energy from the continuum level density of the virtual state. When we assume a negative real energy $E_{v}$ for the virtual pole, the continuum level density of the virtual state is considered to be expressed as

$$
\Delta^{\operatorname{virt}}(E) \propto \frac{1}{E-E_{v}} .
$$

Using numerical data shown in Fig. 8, we can estimate $E_{v} \approx$ $-0.001 \mathrm{MeV}$.

The Jost function method can be easily applied to the present two-body model of Eq. (8) [6], and it is possible to solve virtual states directly. For $V_{1}=-1.42 \mathrm{MeV}$, in the Jost function method, we obtain a solution of the virtual state at $E_{v}=-4.97 \times 10^{-6} \mathrm{MeV}$. Comparing this result, the present $E_{v} \approx-0.001 \mathrm{MeV}$ seems rather large in magnitude. However, the present result should be considered reasonable, because it is difficult for the CSM, where complex eigenvalue problems have to be solved with the basis expansion, to keep a numerical accuracy with around six figures.

\section{SUMMARY AND CONCLUSION}

The photodistintegration cross section for the $1 / 2^{+}$state in ${ }^{9} \mathrm{Be}$ shows a peculiar enhancement near the ${ }^{8} \mathrm{Be}+n$ threshold energy and its origin is interesting in relation with the unbound states of ${ }^{9} \mathrm{Be}$ such as virtual state. In this study, simulating the ${ }^{8} \mathrm{Be}+n$ system, we solved the schematic two-body potential model with the CSM in detail.

In this model calculation, much interest focused on the $s$-wave virtual state because it was discussed as an origin of the peak of the photodisintegration cross section in ${ }^{9} \mathrm{Be}$ in the 
previous work [4]. Another aim of this work is to show that the virtual state can be successfully described in the CSM, which has been believed to be not able to treat such a state.

The two-body potential model was shown to reproduce a peak in the $E 1$ transition strength at a lower energy region by choosing an appropriate value of the potential strength, which has a very similar shape, as was observed in the photodisintegration cross section in ${ }^{9} \mathrm{Be}$. To confirm that its potential strength has a solution of the virtual state, we calculated the continuum level density and the phase shift using eigenvalue solutions in the CSM. From the characteristic phase shift behavior and scattering length, it was concluded that the peak in the $E 1$ transition strength calculated just above the threshold is caused by the virtual state.

In the CSM, the virtual state cannot be obtained as an isolated solution, but the continuum solutions are considered to include the effect of the virtual state. We tried to extract the information of the virtual-state pole in terms of the continuum solutions in the CSM. The virtual-state energy $E_{v}$ obtained using the continuum level density was compared with the solution of the Jost function method [6]. The result of very small energy of the virtual state is consistent with each other when we employ the basis expansion approach with a finite number of basis states. But it is difficult for the CSM to reproduce the energy within high numerical accuracy of the order of $10^{-6}$ as was discussed in Ref. [29].

We here employed the simple potential model, because it is necessary to investigate the properties of virtual states in detail and the reliability of the virtual-state solutions in the CSM as comparing with the solutions of the Jost function method. After confirming their reliability, since the Jost function method is not available for three-body systems such as the $\alpha+\alpha+n$ for ${ }^{9} \mathrm{Be}$, we can study a wide class of unbound states including virtual states in three-body systems in the CSM.

\section{ACKNOWLEDGMENTS}

This work was supported by JSPS KAKENHI Grants No. 25400241 and No.15K05091 and the National University of Mongolia's support for High impact research programm.
[1] H. A. Bethe and P. Morrison, Elementary Nuclear Theory, 2nd ed. (John Wiley \& Sons, New York, 1956), Vol. 80.

[2] I. Tanihata, H. Savajols, and R. Kanungo, Prog. Part. Nucl. Phys. 68, 215 (2013).

[3] T. Sasaqui, K. T. Kajino, G. Mathews, K. Otsuki, and T. Nakamura, Astrophys. J. 634, 1173 (2005).

[4] M. Odsuren, Y. Kikuchi, T. Myo, M. Aikawa, and K. Katō, Phys. Rev. C 92, 014322 (2015).

[5] N. Tanaka, Y. Suzuki, K. Varga, and R. G. Lovas, Phys. Rev. C 59, 1391 (1999).

[6] H. Masui, S. Aoyama, T. Myo, K. Katō, and K. Ikeda, Nucl. Phys. A 673, 207 (2000).

[7] Y. K. Ho, Phys. Rep. 99, 1 (1983).

[8] N. Moiseyev, Phys. Rep. 302, 211 (1998).

[9] S. Aoyama, T. Myo, K. Katō, and K. Ikeda, Prog. Theor. Phys. 116, 1 (2006).

[10] T. Myo, Y. Kikuchi, H. Masui, and K. Katō, Prog. Part. Nucl. Phys. 79, 1 (2014).

[11] T. Myo, K. Katō, S. Aoyama, and K. Ikeda, Phys. Rev. C 63, 054313 (2001).

[12] T. Myo, K. Katō, H. Toki, and K. Ikeda, Phys. Rev. C 76, 024305 (2007).

[13] T. Myo, R. Ando and K. Katō, Phys. Lett. B 691, 150 (2010).

[14] T. Myo, Y. Kikuchi and K. Katō, Phys. Rev. C 85, 034338 (2012).

[15] H. Horiuchi, K. Ikeda, and K. Katō, Prog. Theor. Phys. Suppl. 192, 1 (2012).
[16] Y. Kikuchi, T. Myo, K. Katō, and K. Ikeda, Phys. Rev. C 87, 034606 (2013).

[17] S. Ohtsubo, Y. Fukushima, M. Kamimura, and E. Hiyama, Prog. Theor. Exp. Phys. 2013, 073 D02 (2013).

[18] A. Csótó, B. Gyarmati, A. T. Kruppa, K. F. Pál, and N. Moiseyev, Phys. Rev. A 41, 3469 (1990).

[19] J. Aguilar and J. M. Combes, Commun. Math. Phys. 22, 269 (1971); E. Balslev and J. M. Combes, ibid. 22, 280 (1971).

[20] R. D. Levine, Quantum Mechanics of Molecular Rate Processes (Clarendon Press, Oxford, 1969), Vol. 101.

[21] S. Shlomo, Nucl. Phys. A 539, 17 (1992).

[22] T. Myo and K. Katō, Prog. Theor. Phys. 98, 1275 (1997).

[23] B. G. Giraud and K. Katō, Ann. Phys. 308, 115 (2003).

[24] B. G. Giraud, K. Katō, and A. Ohnishi, J. Phys. A 37, 11575 (2004).

[25] R. Suzuki, T. Myo, and K. Katō, Prog. Theor. Phys. 113, 1273 (2005).

[26] M. Odsuren, K. Katō, M. Aikawa, and T. Myo, Phys. Rev. C 89, 034322 (2014).

[27] J. R. Taylor, Scattering Theory (John Wiley \& Sons, New York, 1972).

[28] E. Hiyama, Y. Kino, and M. Kamimura, Prog. Part. Nucl. Phys. 51, 223 (2003).

[29] A. T. Kruppa and K. Katō, Prog. Theor. Phys. 84, 1145 (1990). 